PROCEEDINGS OF THE

AMERICAN MATHEMATICAL SOCIETY

Volume 133, Number 2, Pages 431-436

S 0002-9939(04)07707-X

Article electronically published on September 16, 2004

\title{
INFINITE TIME BLOW-UP FOR SUPERLINEAR PARABOLIC PROBLEMS WITH LOCALIZED REACTION
}

\author{
PHILIPPE SOUPLET
}

(Communicated by David S. Tartakoff)

Abstract. We consider the nonlocal diffusion equation

$$
u_{t}-u_{x x}=u^{p}\left(t, x_{0}(t)\right),
$$

on the space interval $(0,1)$, with Dirichlet boundary conditions. It is known that if the curve $x_{0}(t)$ remains in a compact subset of $(0,1)$ for all times, then blow-up cannot occur in infinite time. The aim of this paper is to show that the assumption on $x_{0}$ is sharp: for a large class of functions $x_{0}(t)$ approaching the boundary as $t \rightarrow \infty$, blow-up in infinite time does occur for certain initial data. Moreover, the asymptotic behavior of the corresponding solution is precisely estimated and more general nonlinearities are also considered.

\section{INTRODUCTION AND MAIN RESULTS}

In this note, we are interested in the following nonlocal parabolic problem:

$$
\left\{\begin{array}{l}
u_{t}-u_{x x}=u^{p}\left(t, x_{0}(t)\right), \quad t>0, \quad 0<x<1, \\
u(t, 0)=u(t, 1)=0, \quad t>0 \\
u(0, x)=u_{0}(x), \quad 0<x<1
\end{array}\right.
$$

Here $p>1, x_{0}:[0, \infty) \rightarrow(0,1)$ is a locally Hölder continuous function, and $u_{0} \in C_{0}([0,1]), u_{0} \geq 0$. This problem, often referred to as diffusion with localized reaction, can be viewed as a simple model for a reaction-diffusion process where the reaction is driven by the temperature or the density at a single site. It is well known that (1.1) admits a unique maximal classical solution $u \geq 0$, whose existence time will be denoted by $T^{*}=T^{*}\left(u_{0}\right) \leq \infty$. For each solution, one of the following three possibilities occurs:

(I) $u$ blows up in finite time in the sup norm:

$$
T^{*}<\infty \quad \text { and } \quad \lim _{t \rightarrow T^{*}}|u(t, .)|_{\infty}=\infty ;
$$

(II) $u$ is global and bounded:

$$
T^{*}=\infty \quad \text { and } \quad \sup _{t \geq 0}|u(t, .)|_{\infty}<\infty
$$

Received by the editors December 4, 2002.

2000 Mathematics Subject Classification. Primary 35K60, 35B40.

Key words and phrases. Semilinear diffusion equation, localized reaction, nonlocal parabolic problem, blow-up in infinite time, asymptotic behavior. 
(III) $u$ is global and unbounded:

$$
T^{*}=\infty \quad \text { and } \quad \limsup _{t \rightarrow \infty}|u(t, .)|_{\infty}=\infty .
$$

This problem and its higher-dimensional analogue (with the Laplace operator) was studied in [1], [3], [1], [19]-[21]. Other nonlinearities, for example the exponential, were also considered in some of these papers. In particular, it is known for (1.1) that (I) occurs if $u_{0}$ is suitably large and that (II) occurs if $\left|u_{0}\right|_{\infty}$ is sufficiently small. The asymptotic behavior of solutions blowing up in finite time was also studied in these papers. For results on finite time blow-up concerning semilinear systems or degenerate equations with such reactions terms, we refer to [4, 9, 14,15$]$.

On the other hand, it was recently proved in 18 that if

$$
x_{0}:[0, \infty) \rightarrow K, \quad \text { where } K \text { is a compact subset of }(0,1),
$$

then (III) can never occur. The necessity of the assumption (1.2) was left as an open question. The aim of the present paper is to answer this question positively by constructing a wide class of functions $x_{0}$ violating (1.2) and such that (III) does occur for certain $u_{0}$.

Theorem 1. For all $p>1$, there exist $x_{0}:[0, \infty) \rightarrow(0,1)$ of class $C^{1}$ and $u_{0} \in C_{0}([0,1]), u_{0} \geq 0$, such that

$$
\begin{gathered}
\lim _{t \rightarrow \infty} x_{0}(t)=0, \\
T^{*}\left(u_{0}\right)=\infty \quad \text { and } \quad \lim _{t \rightarrow \infty}|u(t, .)|_{\infty}=\infty .
\end{gathered}
$$

Although we are still unable to prove that (III) occurs for every function $x_{0}(t)$ converging to 0 as $t \rightarrow \infty$, our next result shows that we may prescribe essentially any order of decay of $x_{0}$ that is not faster than exponential. Moreover, we describe the asymptotic behavior of the unbounded global solution that we construct. Hereafter, for positive functions $f, g$, we write $f \sim g$ if $c^{-1} \leq f(t) / g(t) \leq c$ as $t \rightarrow \infty$ for some constant $c>0$.

Proposition 2. Let $\varepsilon:[0, \infty) \rightarrow(0, \infty)$ be $C^{1}$ and assume that, for some $C>0$,

$$
\lim _{t \rightarrow \infty} \varepsilon(t)=0 \quad \text { and } \quad-C \varepsilon(t) \leq \varepsilon^{\prime}(t) \leq 0 \quad \text { as } t \rightarrow \infty .
$$

Then there exists a function $x_{0}$ for which Theorem 1 is satisfied and such that

$$
x_{0}(t) \sim \varepsilon(t), \quad \text { as } t \rightarrow \infty .
$$

Moreover, the solution $u$ of (1.1) constructed in Theorem 1 satisfies

$$
|u(t)|_{\infty} \sim \varepsilon^{-\frac{p}{p-1}}(t), \quad \text { as } t \rightarrow \infty
$$

and, for some $C_{1}, C_{2}>0$,

$$
C_{1}|u(t)|_{\infty} \sin (\pi x) \leq u(t, x) \leq C_{2}|u(t)|_{\infty} \sin (\pi x), \quad t \geq 0, \quad 0<x<1 .
$$

Remark 1.1. Let us point out that for the coresponding problem with local reaction term:

$$
u_{t}-\Delta u=u^{p}
$$

with Dirichlet boundary conditions, the question of boundedness of global solutions has been the subject of intensive investigation (see [2], [6]-[8], [12], [16] and the references therein, and [5, 17] for recent surveys). In particular, unlike for (1.1), 
infinite time blow-up never occurs for (1.3) in space dimension 1 (or 2, but it can occur in 3 or more dimensions).

In the next section, instead of the power nonlinearity, we consider general nonlinearities $F$ with arbitrary growth and, under mild assumptions, we show that infinite time blow-up still occurs (see Theorem 2.1 below). Theorem 1 and Proposition 2 will be derived as consequences of Theorem 2.1.

The idea of our proof is indirect. We first solve a linear heat equation, with a prescribed, spatially constant, right-hand side $h(t)$, which grows to infinity as $t \rightarrow \infty$, and we establish some sharp estimates on the solution $v$ of this problem. These estimates enable us to show that $h(t)$ may be written as $F\left(v\left(t, x_{0}(t)\right)\right)$ for a suitable $x_{0}(t)\left(C^{1}\right.$ in $\left.t\right)$, that we determine in terms of $h$ and $v$ and that we may then estimate.

\section{More General Results AND PROOFs}

Consider the more general problem

$$
\left\{\begin{array}{l}
u_{t}-u_{x x}=F\left(u\left(t, x_{0}(t)\right)\right), \quad t>0, \quad 0<x<1, \\
u(t, 0)=u(t, 1)=0, \quad t>0, \\
u(0, x)=u_{0}(x), \quad 0<x<1,
\end{array}\right.
$$

where $F:[0, \infty) \rightarrow[0, \infty)$ is of class $C^{1}$. We shall prove:

Theorem 2.1. Assume

$$
\lim _{s \rightarrow \infty} \frac{F(s)}{s}=\infty \quad \text { and } \quad F^{\prime}(s)>0 \text { for } s>0 \text { large. }
$$

Let $M:[0, \infty) \rightarrow(0, \infty)$ of class $C^{1}$ satisfy

$$
\lim _{s \rightarrow \infty} M(s)=\infty
$$

and, for some $C, s_{0}>0$,

$$
0 \leq M^{\prime}(s) \leq C M(s), \quad s \geq s_{0} .
$$

Then there exist $x_{0}:[0, \infty) \rightarrow(0,1)$ of class $C^{1}$ and $u_{0} \in C_{0}([0,1]), u_{0} \geq 0$, such that the solution $u$ of problem (2.1) satisfies

$$
\begin{aligned}
T^{*}\left(u_{0}\right)=\infty, \quad|u(t, .)|_{\infty} \sim M(t) \quad \text { as } t \rightarrow \infty, \\
C_{1} M(t) \sin (\pi x) \leq u(t, x) \leq C_{2} M(t) \sin (\pi x), \quad t \geq 0, \quad 0<x<1
\end{aligned}
$$

for some $C_{1}, C_{2}>0$ and, for some $K>0$,

$$
x_{0}(t) \sim \frac{F^{-1}(M(t+K))}{M(t+K)} \text { as } t \rightarrow \infty .
$$

Remark 2.1. If, moreover, we assume for instance that $F(t) / t^{a}$ is nondecreasing for $t$ large and for some $a>0$, then, actually,

$$
x_{0}(t) \sim \frac{F^{-1}(M(t))}{M(t)} \text { as } t \rightarrow \infty .
$$

Remark 2.2. The result of [18] on boundedness of global solutions under the assumption (1.2) applies to any nondecreasing $F$ satisfying a superlinearity assumption. This assumption (1.2) is thus sharp also for general $F$. 
In what follows, we put $\varphi(x)=\sin (\pi x)$ and $\lambda=\pi^{2}$. For the proof of Theorem 2.1, we prepare the following lemma.

Lemma 2.2. Assume that $g:[0, \infty) \rightarrow(0, \infty)$ of class $C^{1}$ satisfies

$$
0 \leq g^{\prime}(s) \leq C g(s), \quad s \geq K,
$$

for some $C>0$ and $K \geq 0$. Let $\mu>0$, and let $v(t, x)$ be the solution of

$$
\left\{\begin{array}{l}
v_{t}-v_{x x}=g(t+K), \quad t>0, \quad 0<x<1, \\
v(t, 0)=v(t, 1)=0, \quad t>0 \\
v(0, x)=\mu \varphi(x), \quad 0<x<1
\end{array}\right.
$$

Then $v$ satisfies

$$
\begin{aligned}
C_{1} g(t+K) \varphi(x) & \leq v(t, x) \\
& \leq C_{2} g(t+K) \varphi(x), \quad t \geq 0, \quad 0<x<1,
\end{aligned}
$$

with $C_{1}=\widetilde{C}_{1} \min \left(1, \frac{\mu}{g(K+1)}\right), C_{2}=\widetilde{C}_{2}\left(1+\frac{\mu}{g(K)}\right)$, where $\widetilde{C}_{1}, \widetilde{C}_{2}>0$ depend only on $g$ (not on $K, \mu)$.

Proof. Denote by $(S(t))_{t \geq 0}$ the Dirichlet heat semigroup on the interval $(0,1)$, by $\chi$ the characteristic function of the interval $(0,1)$, and set $h(t)=g(t+K)$. We have

$$
v(t, x)=\mu S(t) \varphi+\int_{0}^{t} h(s) S(t-s) \chi d s, \quad t \geq 0 .
$$

Let us first prove the lower estimate. It is clear that

$$
S(t) \chi \geq S(t) \varphi=e^{-\lambda t} \varphi, \quad t \geq 0 .
$$

On the other hand, by assumption, we have $\left(h(s) e^{-C s}\right)^{\prime}=\left(h^{\prime}-C h\right) e^{-C s} \leq 0$ for $s \geq 0$, so that

$$
h(s) \geq e^{-C} h(t) \quad \text { for all } t \geq 1 \text { and all } s \in[t-1, t] .
$$

Assume $t \geq 1$. Using (2.11), (2.12) and (2.13), we obtain

$$
\begin{aligned}
v(t, x) & \geq \int_{t-1}^{t} h(s) S(t-s) \varphi d s \\
& \geq\left(\int_{t-1}^{t} h(s) e^{-\lambda(t-s)} d s\right) \varphi(x) \geq e^{-(\lambda+C)} h(t) \varphi(x) .
\end{aligned}
$$

If $t \in(0,1)$, we just note that

$$
v(t, x) \geq \mu e^{-\lambda} \varphi(x) \geq \frac{\mu e^{-\lambda}}{g(K+1)} h(t) \varphi(x)
$$

and the lower estimate follows.

Let us turn to the upper estimate. It is known (see e.g. [10]) that

$$
S(s) \chi \leq c s^{-1 / 2} \varphi(x), \quad s \in(0,1] .
$$

Also, as a consequence, it follows that

$$
S(s) \chi \leq c S(s-1) \varphi \leq c e^{-\lambda(s-1)} \varphi(x), \quad s \geq 1 .
$$


Since $g$ is nondecreasing, we deduce from (2.11), (2.14) and (2.15) that

$$
\begin{aligned}
v(t, x) & \leq \mu S(t) \varphi+h(t) \int_{0}^{t} S(s) \chi d s \\
& \leq\left[\mu e^{-\lambda t}+c h(t)\left(\int_{0}^{1} s^{-1 / 2} d s+1_{\{t>1\}} \int_{1}^{t} e^{-\lambda(s-1)} d s\right)\right] \varphi(x) \\
& \leq\left(\frac{\mu}{g(K)}+c\left(2+\lambda^{-1}\right)\right) h(t) \varphi(x),
\end{aligned}
$$

whence the upper estimate follows.

Proof of Theorem 2.1. Put $g(t)=M(t)$. For all $K$ sufficiently large, $g$ satisfies (2.9) and, due to $(2.2),(2.3)$, it follows that $g(K)>F(0)$ and

$$
F^{\prime}(s)>0, \quad \text { for any } s \text { such that } F(s) \geq g(K) \text {. }
$$

Let $v$ be the solution of (2.10) with the choice $\mu=g(K+1)$. Since $\varphi_{x}>0$ in $[0,1 / 2)$, it is easily seen that

$$
v_{x}(t, x)>0 \text { in }[0, \infty) \times[0,1 / 2) .
$$

Let us first show that for all $t \geq 0$,

$$
\text { there exists } x_{0}=x_{0}(t) \in(0,1 / 2) \text { such that } g(t+K)=F\left(v\left(t, x_{0}(t)\right)\right) \text {. }
$$

This will imply that $u:=v$ is a (global unbounded) solution of (2.1). By the lower estimate in Lemma 2.2, we have $v(t, 1 / 2) \geq \widetilde{C}_{1} g(t+K)$. Recall that $\widetilde{C}_{1}$ depends only on $g$, not on $K, \mu$. Due to $(2.2)$, it follows that for $K \geq K_{0}(F, g)$ sufficiently large,

$$
F(v(t, 1 / 2)) \geq F\left(\widetilde{C}_{1} g(t+K)\right)>g(t+K), \quad t \geq 0 .
$$

Since $g(t+K) \geq g(K)>F(0)=F(v(t, 0))$, (2.18) follows immediately. Moreover, due to (2.16) and (2.17), $x_{0}(t)$ is actually unique and the Implicit Function Theorem implies that $x_{0}(t)$ is a $C^{1}$ function.

The assumption (2.4) implies that $g(t+K) \sim g(t)$ as $t \rightarrow \infty$ (cf. the proof of (2.13)). Since $|v(t)|_{\infty} \sim g(t+K)$ by Lemma 2.2, we get (2.5) and (2.6). Let us prove the estimate $(2.7)$. Noting that $F^{-1}$ is well-defined on $[g(K), \infty)$, we have $v\left(t, x_{0}(t)\right)=F^{-1}(g(t+K))$ for all $t \geq 0$. Lemma 2.2 thus implies

$$
\frac{F^{-1}(g(t+K))}{C_{2} g(t+K)} \leq \varphi\left(x_{0}(t)\right) \leq \frac{F^{-1}(g(t+K))}{C_{1} g(t+K)}, \quad t \geq 0,
$$

hence (2.7).

Finally, under the assumption in Remark $2.1, s^{-1 / a} F^{-1}(s)$ is nonincreasing for $s$ large. Since $F^{-1}$ is increasing and $g(t+K) \leq C^{\prime} g(t)$, we have, for some $C_{3} \geq$ $1, F^{-1}(g(t+K)) \leq F^{-1}\left(C_{3} g(t)\right) \leq C_{3}^{1 / a} F^{-1}(g(t))$ for large $t$ and (2.8) follows easily.

Proof of Theorem 1 and Proposition 2. This is an immediate consequence of Theorem 2.1 and Remark 2.1, by setting $M(t):=\varepsilon^{-\frac{p}{p-1}}(t)$ (note that the assumption on $\varepsilon$ in Proposition 2 implies that $M(t)$ fulfills the assumptions in Theorem 2.1).

Remark 2.3. The hypotheses on $g$ in Lemma 2.2 and on $F, M$ in Theorem 2.1 might still be slightly weakened, but we have refrained from doing this for simplicity. 


\section{REFERENCES}

[1] J. R. Cannon, H.-M. Yin, A class of nonlinear non-classical parabolic equations, J. Differ. Equations, 79 (1989), 266-288. MR1000690|(90f:35104)

[2] T. Cazenave, P.-L. Lions, Solutions globales d'équations de la chaleur semi linéaires, Commun. Partial Differ. Equations, 9 (1984), 955-978. MR0755928 (87k:35125)

[3] J. M. Chadam, A. Peirce, H.-M. Yin, The blow-up property of solutions to some diffusion equations with localized nonlinear reactions, J. Math. Anal. Appl., 169 (1992), 313-328. MR.1180894 (93h:35092)

[4] W. Deng, Z. Duan, C. XIE, The blow-up rate for a degenerate parabolic equation with a non-local source, J. Math. Anal. Appl., 264 (2001), 577-597. MR.1876751(2002i:35107)

[5] M. FILA, Boundedness of global solutions of nonlinear parabolic problems, Proceedings of the 4th European Conference on Elliptic and Parabolic Problems (Rolduc, June 2001), pp. 88-102, World Scientific, 2002. MR1937530 (2003i:35130)

[6] M. Fila, Ph. Souplet, F. Weissler, Linear and nonlinear heat equations in $L_{\delta}^{q}$ spaces and universal bounds for global solutions, Math. Annalen, 320 (2001), 87-113. MR1835063 (2002j:35149)

[7] V. A. Galaktionov, J. L. VÁzquez, Continuation of blow-up solutions of nonlinear heat equations in several space dimensions, Comm. Pure Appl. Math., 50 (1997), 1-67. MR.1423231 (97h:35085)

[8] Y. Giga, A bound for global solutions of semilinear heat equations, Commun. Math. Phys., 103 (1986), 415-421. MR0832917|(87j:35187)

[9] Z. Lin, C. XIE, M. WANG, The blow-up properties of solutions to a parabolic system with localized nonlinear reactions, Acta Math. Sci., 18 (1998), 413-420. MR.1696579|(2000b:35114)

[10] Y. Martel, Ph. Souplet, Small time boundary behavior of solutions of parabolic equations with noncompatible data, J. Math. Pures Appl., 79 (2000), 603-632. MR1770663 (2001j:35124)

[11] I.-H. NAM, H.-K. JU, Asymptotic behavior of blow-up solution of a localized semilinear parabolic equation, Honam Math. J., 19 (1997), 61-69. MR1460353 (98h:35126)

[12] W.-M. Ni, P. E. SACKs, J. Tavantzis, On the asymptotic behavior of solutions of certain quasilinear parabolic equations, J. Differ. Equations, 54 (1984), 97-120. MR0756548 $(87 \mathrm{j}: 35057)$

[13] A. OKADA, I. FUKUDA, Blow-up of solutions of nonlinear parabolic equations with localized reaction. in: Free boundary problems: theory and applications, II (Chiba, 1999), 358366, GAKUTO Internat. Ser. Math. Sci. Appl., 14, Gakkōtosho, Tokyo, 2000. MR1794365 (2001i:35158)

[14] M. Pedersen, Z. Lin, Coupled diffusion systems with localized nonlinear reactions, Comput. Math. Appl., 42 (2001), 807-816. MR1846188(2002e:35133)

[15] M. Pedersen, Z. Lin, The profile near blowup time for solutions of diffusion systems coupled with localized nonlinear reactions, Nonlinear Anal., 50 (2002), 1013-1024. MR 1915320 (2003i:35160)

[16] P. QUitTNeR, Universal bound for global positive solutions of a superlinear parabolic problem, Math. Annalen, 320 (2001), 299-305. MR.1839765 (2002f:35130)

[17] P. Quittner, A priori bounds for solutions of parabolic problems and applications, Math. Bohemica, 127 (2002), 329-341. MR 1981537 (2004d:35120)

[18] P. Rouchon, Boundedness of global solutions for nonlinear diffusion equations with localized source, Differ. Integral Equations, 16 (2003), 1083-1092. MR1989542 (2004d:35134)

[19] Рн. Souplet, Blow-up in nonlocal reaction-diffusion equations, SIAM J. Math. Anal., 29 (1998), 1301-1334. MR1638054 (99h:35104)

[20] Ph. Souplet, Uniform blow-up profiles and boundary behavior for diffusion equations with nonlocal nonlinear source, J. Differ. Equations, 153 (1999), 374-406. MR 1683627 (2000e:35105)

[21] L. WANG, Q. Chen, The asymptotic behavior of blowup solution of localized nonlinear equation, J. Math. Anal. Appl., 200 (1996), 315-321. MR.1391152 (97d:35096)

Département de Mathématiques, insset Université de Picardie, 02109 St-Quentin, France - ANd - Laboratoire de Mathématiques Appliquées, UMR CNRS 7641, Université de Versailles, 45 avenue des États-Unis, 78035 Versailles, France

E-mail address: souplet@math.uvsq.fr 